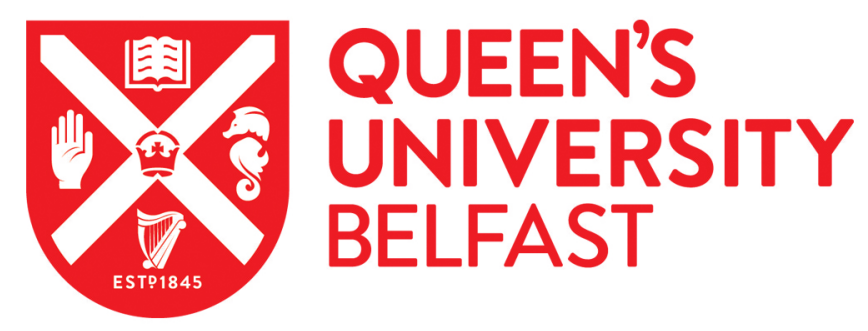

\title{
Challenges of conducting research with young offenders with traumatic brain injury
}

O'Rourke, C., Templeton, M., Cohen, M., \& Linden, M. (2018). Challenges of conducting research with young offenders with traumatic brain injury. Journal of Head Trauma Rehabilitation.

https://doi.org/10.1097/HTR.0000000000000392

Published in:

Journal of Head Trauma Rehabilitation

Document Version:

Peer reviewed version

Queen's University Belfast - Research Portal:

Link to publication record in Queen's University Belfast Research Portal

Publisher rights

Copyright $\odot 2018$ Wolters Kluwer Health This work is made available online in accordance with the publisher's policies. Please refer to any applicable terms of use of the publisher.

\section{General rights}

Copyright for the publications made accessible via the Queen's University Belfast Research Portal is retained by the author(s) and / or other copyright owners and it is a condition of accessing these publications that users recognise and abide by the legal requirements associated with these rights.

Take down policy

The Research Portal is Queen's institutional repository that provides access to Queen's research output. Every effort has been made to ensure that content in the Research Portal does not infringe any person's rights, or applicable UK laws. If you discover content in the Research Portal that you believe breaches copyright or violates any law, please contact openaccess@qub.ac.uk. 
2 Conall O'Rourke ${ }^{1}$, Michelle Templeton ${ }^{1}$, Miriam H Cohen ${ }^{2,3}$, Mark A. Linden ${ }^{1}$

3

$4 \quad{ }^{1}$ School of Nursing and Midwifery, Queen's University Belfast, Belfast, United Kingdom

$5{ }^{2}$ Department of Psychology, University of Exeter, UK

$6 \quad{ }^{3}$ UK Centre for Tobacco and Alcohol Studies, University of Bristol, UK

7

8

Introduction

9 A growing body of research has developed around traumatic brain injury (TBI) among offender

10 populations. Rates of $\mathrm{TBI}$ amongst these populations are reported as substantially higher than those

of the general population (1). Many of the neuropsychological outcomes of TBI (e.g. aggression, impulsivity, disinhibition, and self-regulation) may place an individual at significant disadvantage when dealing with the criminal justice system. More recently, it has been suggested that a causal link may exist between TBI and offending, though the accuracy and complexity of this link is unknown (2). Though many studies in this area utilise populations of offenders, few offer critical reflections on the complexity and challenges of working with such groups. Drawn from first-hand experience of conducting research within prisons, the aim of this article is twofold; to highlight the issues researchers and clinicians can face when working with these populations, and provide critical considerations for future research conducted in this area.

\section{Heterogeneity in offending}

21 Recruiting from prison populations requires acknowledgment of the differences between, for example, individuals who are incarcerated for drug offences versus sexual offences. Both crimes incorporate very different behaviours which may be guided by different motivations and circumstances. There is a tendency for TBI studies in offending populations to combine different criminal convictions without acknowledging or running sub-group analyses based on these. In order to help reduce heterogeneity, future studies should consider limiting recruitment to those convicted of similar crimes, or aim to collect suitably sized samples to enable differentiation between types of criminal behaviour. Further to this, in attempting to draw conclusions about criminal behaviours using prison population data, it is 
important to note that the composition of and fluctuations in prison populations over time does not necessarily reflect wider crime rates. Prison populations are highly sensitive to changes in sentencing and enforcement. One such example is the rise in custodial sentences for sexual offences in the UK (3). While the number of offenders serving sentences for sexual offences remained largely stable between 1993 and 2004, the introduction of the Sexual Offences Act 2003 saw a steady and continuing rise in the number of sexual offence convictions (3). This is complicated further when considering the myriad of factors that can influence custodial sentencing decisions. Quality of legal representation, race (4), socioeconomic background (5), and gender can impact the composition of a sample, with males, for example, much more likely to receive an immediate custodial sentence than females (6).

\section{Complexity of Need}

Examinations of offender groups often fail to acknowledge the highly complex needs of this population. There is a tendency in research to overlook the broader behavioural, health, and social difficulties experienced by this vulnerable group. Work by the Prison Reform Trust (2016) highlighted physical and mental disability, depression, anxiety, and psychosis, alongside severe drug and alcohol abuse among the challenges experienced by offenders in the UK (7). Significantly high rates of dualdiagnoses require particular consideration, with Pycroft and Green (2016) noting the unique and often unaddressed needs of this group (8).

Such complexity poses issues of both an ethical and practical nature. Researchers must consider whether their study would be appropriate for a young offender if such issues are present. Indications of disability or disorder should be taken into account when considering eligibility for participation, although it is important not to make presumptions about an individual's capacity based on these. This should also be considered in relation to capacity to give informed consent, ensuring that individuals fully understand what is involved and have been given an opportunity to consider and ask questions about the research. Comprehension should be checked before formally taking consent (for example, by questioning the young person on aspects of the information sheet or by asking them to describe the research process). 
56 This should not only be considered in relation to informed consent, but also quality of data collected.

57 Psychometric assessments which contain statements, vocabulary, or phrasing unfamiliar to the 58 individual may be compromised if task comprehension is poor. This is equally important when aiming 59 to encapsulate offenders' voices and experiences (11). Researchers may have differing views on 60 offenders' ability to participate and express an opinion, and should be conscious as to whether their 61 approach is tailored to this population. While critical analysis of research tools and methods is 62 required, acknowledging these individuals as active agents, capable of understanding, influencing and 63 directing research (12) is equally important.

64 Due consideration must be given not only to an individuals' capacity to consent but to ensuring they have a clear understanding of their role in the research. This consideration also relates to section 4 of the British Psychological Society (BPS) Code of Human Research Ethics (9). It is essential to make clear that the study is separate to the work conducted by criminal justice services. It must be emphasised that participation is voluntary and will not impact upon their sentence or affect privileges for good behaviour. Prison staff and researchers can be in a position of real or perceived authority and the potential for coercion should be recognised and addressed. This is true for prisoners and young people generally, but additional precautions should be taken when working with members of these populations with a TBI, given their heightened vulnerability.

73 Another aspect to consider, especially when collecting sensitive data regarding head injury history, substance use, mental health issues and early adversity is the personal and sometimes upsetting nature of these measures. Participants should be made aware of the sensitive nature of such questions prior to their administration, and understand that they can choose to omit those which make them uncomfortable. Procedures and pathways should be in place in the event participants do experience distress as a result of such questioning. If a young person discloses information indicating an intention to harm themselves or others, it is vital that a thorough protocol is in place to breach confidentiality and inform all necessary parties (10). Any doubts regarding these should be discussed with appropriate criminal justice staff who are familiar with the young person. 
83 Cognitive and learning difficulties may call into question the reliability of offenders' responses,

84 particularly in the context of self-report health measures. Despite this concern, and the high number of

85 self-report based studies in this area, little research exists examining the reliability of offenders as research participants. Bai and colleagues (2014) noted the difficulty in obtaining objective sources of

87 data for this group, with medical records often limited by illegibility or incomplete documentation (13). Work by Schofield and colleagues (2011) examining the reliability of offenders in reporting TBI when compared to medical records, concluded that offender self-report was generally accurate (14). The authors additionally acknowledge that medical records are themselves often incomplete and may not include information on the Glasgow Coma Scale (GCS), loss of consciousness duration (LOC), or even record that a TBI has occurred. More recently, McKinlay and Horwood (2017) showed adults to have relatively low accuracy of recall for childhood mild TBI events, with $9 \%$ recalled between the ages of 0-4 years and $31 \%$ between 5-9 years (15). With such paediatric events showing low rates of adult recall, it is possible that many of the most significant injuries in childhood remain hidden from adult self-report.

In the context of offenders, both medical records and self-report measures are susceptible to inaccuracy and likely present with disparate prevalence rates. Nonetheless, researchers in the area must consider both the comparability and usefulness of their collected TBI data. Single question assessments of TBI, or exclusion of injuries without LOC offer limited information to the reader, and thus limited usefulness when comparing datasets. Studies examining self-reported TBI among offenders should aim to collect data on; the most severe injury, the earliest injury, the presence of multiple injuries, and where possible, the cause of injury.

\section{The prison environment}

Some of the greatest difficulties within our research arose from the prison system itself. The rigid, controlled nature of prison life meant that interruptions to planned activities were met with frustration from both young people and prison staff. Interviews had to be scheduled around education and work times, with participants often re-arranging or cancelling interviews so as not to miss particular classes. For this reason, flexibility on the part of the researcher is paramount. Having an internal contact within the prison administration greatly helped with difficulties such as security, freedom of movement, and clearance of research materials. The value of the research to prison services should also be made 
112 clear from the outset and it is important to develop relationships with all levels of prison staff to ensure

113 successful uptake. It is also important that staff have a clear understanding of the purpose of the

114 research and are allowed some influence on how it is delivered. As with any large institution, while some individuals will see value in the work, others will be more sceptical. It is often helpful and effective to give a presentation on the topic of the research prior to study commencement with training on the procedures of the study and a discussion or focus group regarding study logistics. Ideally staff, and where possible, offenders, should have input into the design and conduct of the research. This will encourage staff engagement with the research, which is vital. We strongly advise running a structured feasibility study or pilot prior to the definitive full research study. This could also be supplemented with qualitative staff and participant interviews regarding the acceptability of the research study and an opportunity to feedback on any encountered issues. For an example of one our current feasibility studies and details of the procedural plan, see the following pre-registered protocol (https://osf.io/u29qq/).

\section{Research 'burden'}

Many services within the criminal justice system are strained and experience substantial cut-backs to funding, resulting in staff and service shortages. Research is often a lengthy process, and whilst our work has received support (and often enthusiasm) from criminal justice staff, these individuals often have very limited time to conduct their core duties. Involvement in research can place strain on available staff and resources, putting them at risk of becoming overburdened. It is the responsibility of the researcher to manage the scale of the study and to adjust data collection procedures, or if necessary, discontinue the research effort if they perceive this to be burdensome.

The demands upon researchers within these environments should also be considered. Prisons and criminal justice services are difficult environments within which to conduct research and the nature of TBI research often requires collecting personal and sensitive data. This information can be of a distressing nature, with earlier injuries commonly arising through assault or abuse. Research staff need to be suitably trained in safety procedures, well supported by members of a wider research team, and have the opportunity for supervision regarding more difficult cases. Due to the aforementioned heterogeneity surrounding these populations, large-scale projects are essential, yet these types of studies are often conducted by small teams, or independent researchers. This puts 
pressure on the researcher to recruit a required number of participants within a restricted timescale, which, given the difficulties engaging members of this population - is not always realistic or possible. Unachievable or unrealistic expectations of sample sizes can lead to additional burden and in some cases, termination of research studies leading to wasted or unreported research effort. We should address this in future by encouraging collaboration between institutions and the development of largescale, multi-centre research projects with a supportive network of researchers and expertise.

\section{Conclusions}

Several important factors should be considered when working with young offenders. The diverse and challenging nature of this population poses barriers to the scope and depth of possible investigation. Additionally, though progress is evident, there is a long way to go in terms of fostering a consistent culture of research in this environment. Many of the challenges faced by researchers can be linked to the divergent priorities of researchers and correctional staff. While researchers should reflect on the impact of their presence in this environment, the social context of prison alongside the motivations of offenders as participants also merits consideration.

Implications:

1. Detailed reporting of demographic and criminal history factors are of specific importance given the heterogeneity of this group.

2. Offender groups can present with highly complex, interrelated, and often undiagnosed difficulties and attempts should be made to understand such complexity.

3. The reliability of self-report from offenders is not well known and alternative methods of data collection should be considered.

4. Logistical and bureaucratic challenges mean researchers must be clear with their aims yet flexible in their approach, we encourage conducting pilot and feasibility studies prior to the main study to support this.

5. The social context of the prison environment and group dynamics must be considered when attempting to recruit and work with young offenders.

6. We encourage large-scale, collaborative research efforts when planning future studies involving these complex populations. 
170 1. Shiroma EJ, Ferguson PL, Pickelsimer EE. Prevalence of traumatic brain injury in an offender population: A meta-analysis. J Correct Health Care. 2010;16(2):147-59.

2. Williams H. Traumatic brain injury and crime: causal links and emerging interventions. Dev Med Child Neurol. May;56:6.

3. Ministry of Justice. Story of the Prison Population: 1993 - 2012 England and Wales. 2013. Available from: https://www.gov.uk/government/uploads/system/uploads/attachment_data/file/218185/story-

4. Mitchell TL, Haw RM, Pfeifer JE, Meissner CA. Racial bias in mock juror decision-making: A meta-analytic review of defendant treatment. Law Hum Behav. 2005;29(6):621-37.

5. Devine DJ, Caughlin DE. Do they matter? A meta-analytic investigation of individual characteristics and guilt judgments. Psychol Public Policy, Law. 2014;20(2):109-34.

9. British Psychological Society. Code of Human Research Ethics. 2014. Available from: 
10. British Psychological Society. Code of ethics and conduct. 2009. Available from: http://www.ed.ac.uk/files/atoms/files/bps_code_of_human_research_ethics.pdf

11. Graham A, Powell M, Taylor N, Anderson D, Fitzgerald R. Ethical research involving children. Florence: UNICEF Office of Research-Innocenti. 2013. 2013. Available from: https://aifs.gov.au/publications/family-matters/issue-96/ethical-research-involving-children

12. Handel G, Cahill S, Elkin F. Children and society: The sociology of children and childhood socialization. Roxbury Publishing Company; 2007.

13. Bai JR, Mukherjee DV, Befus M, Apa Z, Lowy FD, Larson EL. Concordance between medical records and interview data in correctional facilities. BMC Med Res Methodol. 2014;14(1):50.

14. Schofield P, Butler T, Hollis S, D'Este C. Are prisoners reliable survey respondents? A validation of self-reported traumatic brain injury (TBI) against hospital medical records. Brain Inj. 2011;25(1):74-82.

15. McKinlay A, Horwood LJ. The accuracy of adult recall for early mild traumatic brain injury. Disabil Rehabil. 2017; 39(13):1296-9.. 